\title{
Why teach Wigner Optics?
}

\section{William Rhodes, Karl-Heinz Brenner, John Sheridan}

William T. Rhodes, Karl-Heinz Brenner, John T. Sheridan, "Why teach Wigner Optics?," Proc. SPIE 9663, Eighth International Topical Meeting on Education and Training in Optics and Photonics, 96630V (6 October 2003); doi: 10.1117/12.2208452

SPIE Event: Eighth International Topical Meeting on Education and Training in Optics and Photonics, 2003, Tucson, Arizona, United States 


\title{
Why teach Wigner Optics?
}

\author{
William T. Rhodes \\ Georgia Institute of Technology, School of Electrical and Computer Engineering, Atlanta, GA, 30332-0250 \\ telephone: +1404-894-2929; fax: +1 404-894-4641; e-mail: wrhodes@ece.gatech.edu \\ Karl-Heinz Brenner \\ Universität Mannheim, Lehrstuhl für Optoelektronik, B6, 23-29, Zi. C 3.11, 68131 Mannheim, Germany \\ telephone: +49621-181-2700; fax:+49621-181-2695; e-mail: brenner@uni-mannheim.de \\ John T. Sheridan \\ University College Dublin, Dept. of Electronic and Electrical Engineering, Belfield, Dublin 4, Republic of Ireland \\ telephone: +353 1-706-1927; fax: +353 1-283-0921; e-mail: john.sheridan@ucd.ie
}

\begin{abstract}
The Wigner distribution function is viewed as an important tool in the analysis of wave progation and image formation. We discuss why it should be taught in optics courses relating to these topics.

(C)2003 Optical Society of America

OCIS codes: (070.2580) Fourier optics; (110.2990) Image formation theory
\end{abstract}

During the past decade or so the Wigner distribution function (WDF) has received increased attention as a tool appropriate for students to use in the analysis of optical wave propagation and imaging systems. Indeed, it has been suggested that "Wigner Optics" replace "Fourier Optics" as the core topic introducing applied physics and engineering students to diffraction theory, imaging theory, and related subjects. As university professors teaching in optics, we would not necessarily go so far as to change a course name. Nevertheless, we believe that the WDF and certain of its applications should definitely be introduced to students of optics. It is the purpose of this paper to explore why? An associated poster paper discusses the issues of what and how?

The introduction of the WDF - which in wave optics expresses in a single function the distribution of the spatial frequency content of a wave field as a function of spatial location - challenges students in part because it results from a dimensionality-increasing transformation: The WDF associated with a 1-D function is a 2-D function; that of a 2-D function is a 4-D function. Simply graphing the WDF of a 2-D complex amplitude distribution confronts one with a near impossibility. It is thus necessary to convince the students (and, of course, the potential instructor) that the WDF of an optical wavefield provides adequately justifying insight into the behavior of that wavefield, even if the wave is modeled only as a 1-D distribution.

The strongest motivation for introducing the WDF into, e.g., a Fourier optics course lies in the utility of the Wigner space-bandwidth diagram (WSBD, sometimes referred to as the Wigner chart), which can provide considerable insight into the effects of lenses and free-space propagation on optical wave fields. The WSBD is simply constructed, being no more than a suitably-dimensioned box, conceptually attached to the WDF associated with the wave field. As the wave propagates, the WSBD undergoes a simple shearing operation in one direction. Passage of the wave through a spherical lens is modeled by a shearing in the orthogonal direction. The shape of the WSBD can give a reasonable estimate of the way energy flows through, e.g., a system of lenses and provide insight into the operation of different kinds of imaging systems. Of special interest is the optical Fourier transform system, which manifests itself through a 90-degree rotation of the WSBD, and its cousin, the optical fractional Fourier transform system, which manifests itself as a rotation of the WSBD through an arbitrary angle. Examples of systems will be presented that provide convincing evidence of the basic method.

Eighth International Topical Meeting on Education and Training in Optics and Photonics,

edited by Barry L. Shoop, Grover Swartzlander Jr., Proc. of SPIE Vol. 9663, 96630V

(C) 2003 SPIE, OSA, ICO · doi: 10.1117/12.2208452 